\title{
Conceptual memory for picture and prose episodes
}

\author{
R. KIM GUENTHER \\ Hamline University, St. Paul, Minnesota 55104
}

\begin{abstract}
In two experiments, subjects decided whether probes were true or false of one of some previously studied picture and prose episodes. The results indicated that response times to probes depicting explicit events were faster when they were in the same modality (picture or prose) as their episodes than when they were in the opposite modality, but response times to probes depicting implicit events were as fast when they were in the opposite modality as when they were in the same modality. These results suggest that the conceptual representations of picture and prose episodes are identical in form. The second experiment indicated that probes could be evaluated as fast from long as from short episodes. This result is consistent with the notion that retrieving information from episodes is done, not by scanning all the events of an episode, but by restricting the search only to the relevant slot in the schema into which the episode is assimilated.
\end{abstract}

Recently, there has been much interest in how meaningful text is represented in memory and how information about text is retrieved from memory (J. R. Anderson, 1976; J. R. Anderson \& Bower, 1973; Kintsch, 1974; Norman \& Rumelhart, 1975; Schank, 1972). One important idea that has emerged from this work is the notion that the conceptual information in a text is stored in memory in an abstract form that preserves little of the physical or linguistic features of the text. Most theories propose that meaning is stored as abstract relations among conceptual symbols, and not as words and sentences. As support for such a claim, a variety of research has shown that subjects in recognition experiments are good at detecting changes of meaning, but not changes in the syntactic or stylistic form of the text (Bransford, Barclay, \& Franks, 1972; Bransford \& Franks, 1971; Sachs, 1967). Other support includes the findings that paraphrases of parts of text can be as effective in aiding recall as verbatim repetitions of some part of the text (R. C. Anderson, 1971; Honeck, 1973), that the truth value of potential inferences from a text can be evaluated as rapidly when the text is written in a syntactically simple form as when it is written in a more complex form (King \& Greeno, 1974; Kintsch \& Monk, 1972), that inferences are often confused with explicitly presented facts (Bransford et al., 1972; Harris \& Monaco, 1978; Johnson, Bransford, \& Solomon, 1973; Potts, 1972; Thorndyke, 1976), and that, under some conditions, the truth value of inferences can be evaluated as rapidly as the truth value of explicitly presented information (Keenan \& Kintsch, 1974; McKoon \& Keenan, 1974).

The experiments reported here investigate another

The author wishes to thank the anonymous reviewers from this journal for their comments on this manuscript. Requests for reprints should be sent to R. Kim Guenther, Department of Psychology, Hamline University, St. Paul, Minnesota 55104. possible implication of the idea that conceptual memory is abstract and preserves little specific linguistic or physical information; this implication is that the conceptual representation of the meaning of a verbal discourse has the same abstract format as the representation of the meaning of a nonverbal, pictorial discourse. That is, while there may be differences between the specific contents of the conceptual representations of prose and pictorial depictions of the prose, the forms of their conceptual representations are identical. Such a hypothesis, labeled here the conceptual identity hypothesis, is not new and has been made or implied by several researchers (J. R. Anderson \& Bower, 1973; Baggett, 1975; Norman \& Rumelhart, 1975; Pezdek, 1977; Pylyshyn, 1973; Rosenberg \& Simon, 1977). However, relatively few studies have demonstrated specifically that the process of evaluating the meaning of discourse (in the sense of understanding implications of the discourse) is identical for picture and prose modalities. An exception is Baggett (1975), who showed that, after a sufficient delay between the presentation of a pictorial discourse and the presentation of test pictures, the truth value of test pictures depicting inferences can be evaluated as rapidly as the truth value of pictures presented explicitly in the discourse, a result similar to that found with verbal discourse (Keenan \& Kintsch, 1974; McKoon \& Keenan, 1974).

The conceptual identity hypothesis was tested in the present research by using a modified version of Baggett's (1975) methodology. Subjects were first presented a series of very short picture and prose episodes depicting stereotypic situations, such as going to a barbershop and robbing a bank, and later were presented probes that depicted events either true or false of one of the previously presented episodes. True probes depicted events that were either explicitly or only implicitly a part of one of the episodes; these true probes were presented 
either in the same or in the opposite modality (picture or prose) as the episode to which they referred. False probes depicted events related to but not implied by any of the episodes; the false probes were also in the same or the opposite modality as the episode to which they were related. Many such false probes depicted events that changed some important feature but were otherwise identical to some explicitly presented event and consistent with the stereotypic situation of their episodes. For example, in a false probe related to a restaurant episode, the server was a waitress rather than the waiter of the original episode. Other false probes depicted events that could have occurred in the context of the stereotypic episode but did not happen to occur in the episode actually presented. For example, in a false probe related to a fishing off the end of a pier episode, the fisherman sat on the end of a pier reading a book. Thus, in order to discriminate implicit and related false probes, subjects were unable to make their true-false decisions by simply determining whether or not the probe fit a simple label of one of the episodes. Rather, subjects were required to base their responses to probes on a conceptual representation that includes information about the specific events that take place in the episode.

The predictions of this experiment require the assumption that the time to make a judgment about the truth value of a probe is, in part, a function of the degree to which information in a probe is stored in a form similar to the memorial representation of the relevant episode. If some set of information in a probe is in a form different from that of its episode, then either that information cannot be recruited into the decision process, which is therefore slowed down, or the information must be translated into a comparable form, and such a translation process takes additional processing time. Given this assumption, it was expected that subjects would judge the truth of an explicitly true probe more rapidly when it was in the same than when it was in the opposite modality of its episode. That is because both the surface or physical features and the conceptual content of explicit probes in the same modality match the surface and conceptual representations of their relevant episodes. However, for explicit probes in the opposite modality, only their conceptual contents match the conceptual representations of the relevant episodes; surface information cannot be recruited into the decision process (Kintsch, 1974). The conceptual identity hypothesis predicts that subjects should judge the truth value of implicit probes in the opposite modality as fast as the truth value of implicit probes in the same modality. Since implicit probes depict events never actually presented in an episode, subjects must decide that such probes are true based on their notions of the episode's meaning and implications-that is, based on the conceptual representation of the relevant episode. According to the conceptual identity hypothesis, this conceptual representation retains no information partic- ular to the stimulus modality or surface qualities of the episode. There are several ways subjects could judge implicit probes. The conceptual representation of a probe might be matched directly to a conceptual fact stored in memory, if that fact was generated during original study. Or, if the inference depicted by a probe is not stored explicitly in the conceptual representation of an episode, subjects may attempt to generate a causal chain between the probe and the episode; if such a chain is easily constructed, the probe may be assumed to be true (Schank, 1975; Thorndyke, 1976). The main tenet of the conceptual identity hypothesis is that, since the form of the conceptual representation of pictorial episodes is identical to that of prose, no translation process is required to convert the conceptual contents of implicit probes in the opposite modality into a form comparable to their episodes' conceptual representations.

The conceptual identity hypothesis may be con. trasted with a conceptual nonidentity hypothesis that would propose that the conceptual representation, although abstract, still retains some features specific to the stimulus modality of the relevant episode. For example, some of the explicit and implicit conceptual information in a pictorial discourse may be stored in the form of visual images (Begg \& Paivio, 1969; Yuille \& Paivio, 1969), whereas the conceptual information in prose is stored in a verbal/lexical form (Hayes-Roth \& Hayes-Roth, 1977). Note that neither a visual image nor a verbal representation need be an exact replica of events in an episode. The conceptual nonidentity hypothesis would predict that subjects should judge the truth of a same-modality implicit probe faster than that of an opposite-modality implicit probe. That is because the conceptual contents of a same-modality implicit probe would closely resemble the form of the conceptual representation of its relevant episode; both conceptual representations would contain the same kind of features particular to their stimulus modality. The conceptual contents of opposite-modality implicit probes, however, would not resemble the form of their episodes' conceptual representations. Therefore, the conceptual contents of the probe would have to be translated into a form comparable to the modality-specific conceptual representations of its episode before the truth value could be determined, and such a translation process would require additional processing time.

Neither the conceptual identity nor the conceptual nonidentity hypothesis makes predictions concerning the decision time difference between explicit and implicit probes. Such a difference would depend crucially on a number of variables not systematically manipulated or controlled for in the present experiments. These variables include the necessity of generating inferences depicted by probes for initial comprehension of an episode, the incentive subjects have to generate inferences during initial comprehension, the number of causal connections required to link an implicit probe to what is 
stored about an episode (i.e., the difficulty of the inference), and the durability of the surface representations of episodes.

\section{EXPERIMENT 1}

\section{Method}

Subjects. The subjects were 32 undergraduates enrolled in the introductory psychology course at Hamline University.

Stimuli. Eight picture and eight corresponding prose episodes were constructed. The eight episodes were all unrelated to each other. These episodes were similar to those used by Baggett (1975) and depicted characters in relatively stereotypic situations, such as going to a barbershop, eating at a restaurant, and making a snowman. The picture episodes were made up of six black-and-white cartoon-like drawings, and their corresponding prose episodes were made up of six simple declarative sentences each sentence was a simple description of its corresponding picture. The pictures and sentences (which will be referred to as events) were presented on slides, one event per slide.

For each episode, two related distractor events were constructed; these represented events related to but not strictly implied by the events in the episodes. Each related distractor event was represented as a picture and as a sentence describing the picture. Generally, related distractors either changed important objects or characters but were otherwise consistent with the stereotypic episode or depicted events that could conceivably occur in the setting of the episode but did not happen to occur in the actually presented episode or that contradicted an inference of an episode. Three of the prose episodes and their related distractors are presented in Table 1.

Finally, a set of 16 unrelated distractor events was constructed. Each unrelated event was represented as a picture and as a sentence describing the picture. The unrelated distractors represented events totally unrelated to any episode.

Apparatus. The apparatus consisted of a Kodak Carousel
AV900 projector, a rear screen, a Marietta digital millisecond timer (Model 14-15-MS), and a response box. The subjects viewed the slides through the rear screen and made their responses by pressing one of two buttons that were in each of the upper corners of the response box. Each subject was allowed to select which of the response buttons would correspond to a "true" response. The box also contained a start button that allowed the subject to initiate a trial during the test phase of the experiment.

Procedure. Subjects were run individually in one 35 -min session that consisted of a practice, study, and test phase. In the practice phase, subjects were presented two episodes (a picture and a prose episode) and 16 probe events based on those episodes. The episodes and probes were stimuli different from those used in the experiment proper, but they had the characteristics of the experimental stimuli.

After the practice phase, subjects began the study phase, in which a set of eight episodes was presented. The entire set was presented twice; the order of the episodes was the same for each presentation. Subjects were instructed to pay close attention to the meaning of the text. For each episode presented in the study phase, two of the six events of each episode were considered filler events; these were presented to all subjects during the study phase but were never used as probes during the test phase. Of the remaining four nonfiller events, two were presented during the study phase and later during the test phase (and became the explicit probes), and the other two were withheld during the study but were presented later during the test phase (and became the implicit probes). A counterbalancing insured that, across subjects, the nonfiller events were presented during the study phase as often as they were withheld from the study phase. Table 1 indicates, for three of the episodes, which events were fillers and which were nonfillers.

Each event in the study phase was presented for $7 \mathrm{sec}$, with an interslide interval of about $1 \mathrm{sec}$, and episodes were separated by a 7 -sec interval, during which the projector projected only light to the rear screen. Each subject saw four picture and four

Table 1

Examples of Prose Stimuli

A man sat down at a table in a restaurant. (filler)

A waiter handed him a menu.*

The man pointed to what he wanted on the menu. (filler)

The waiter brought his food.

The man began to eat his food.*

He finished eating his meal. $\dagger$

A waitress brought his food. (related)

He drank beer at the restaurant. (related)

A bank robber waited in line at the bank. (filler)

The robber got up to the front of the line.

He pulled a gun on the woman teller. (filler)

The teller handed the robber some money.*

The robber put the money into a sack.*

He ran out of the bank with the sack of money. $\dagger$

She held a gun on the robber. (related)

The robber handed the teller some money. (related)

A long haired man approached the entrance of a barbershop. (filler)

The long haired man walked into the barbershop.*

He sat reading a magazine, waiting to get his hair cut. (filler)

The long haired man sat in the barber's chair. $t$

The barber cut his hair.*

The man left the barbershop with shorter hair.

The barber shined the long haired man's shoes. (related)

The man left the barbershop with long hair. (related)

*The explicit events of a short episode for the first half of the subjects in Experiment 2.

tThe explicit events of a short episode for the second half of the subjects in Experiment 2. 
prose episodes; the presentation order of the episodes alternated the modality (picture or prose) of the episodes. For each subject, any given episode was seen only in its picture or in its prose version. A counterbalancing insured that, across subjects, each episode appeared equally often as a picture episode and as a prose episode, and the first episode presented was equally often a picture and a prose episode. Within these constraints, the order of episode presentation was randomized for each subject.

Immediately after the second presentation of all eight episodes, the test phase of the experiment began. The test phase consisted of 64 trials. Subjects initiated each test trial by pressing a start button with their thumb. Approximately $1 \mathrm{sec}$ later, a probe event was projected on the rear screen. Subjects indicated, by pressing a response button with one of their index fingers, if the probe event was in or implied by an episode (a true response) or if the probe event was not implied by any episode (a false response). Reaction time (RT) was measured from the onset of the probe display to the response, which terminated the display. After an interval of about $6 \mathrm{sec}$, during which the experimenter recorded the RT, reset the timer, and advanced the projector, the next trial began. Subjects were instructed to respond as quickly as possible without error and were informed whenever an error was made.

There were four types of probe events that required a true response: (1) an explicitly presented event in the same modality as its episode (explicit same), (2) an explicitly presented event in the modality opposite to that of its episode (explicit opposite), (3) an event withheld during study but implied by an episode and presented in the same modality as its episode (implicit same), and (4) an event withheld during study but implied by an episode and presented in the modality opposite that of its episode (implicit opposite). There were three types of events that required a false response: (1) an event related to an episode and presented in the same modality as its episode (related same), (2) an event related to an episode and presented in the modality opposite to that of its episode (related opposite), and (3) an event unrelated to any episode (unrelated).

Each episode contributed six events to the test phase of the experiment: two explicit events, one presented in the same and one presented in the opposite modality; two implicit events (those withheld during the study phase), one presented in the same and one presented in the opposite modality; and two related events, one presented in the same and one presented in the opposite modality. The remaining 16 probe trials consisted of the 16 unrelated events; 8 were presented as pictures and 8 as sentences.

A counterbalancing was done to insure that, across subjects, each probe event was presented as a picture as often as it was presented as a sentence and each event that required a true response was presented as an explicit event as often as it was presented as an implicit event. Within these constraints, the order of probe events was randomized for each subject.

\section{Results}

The principal data are mean RT milliseconds. The RT analysis includes only correct responses; the mean error rate over subjects was $7.4 \%$, with a range of $3.2 \%$ to $15.7 \%$. In general, errors were positively correlated with RT within each variable. Aberrant RTs, defined as those over 3 standard deviations larger than the overall subject mean RT, were excluded from the data analysis (this exclusion was less than $2 \%$ ), as were data from the practice trials.

Two different analyses of variance were conducted on both the true and false responses. One analysis used RT means computed for each subject and averaged over all episodes and therefore treated subjects as a random factor. The other analysis used RT means computed for each episode and averaged over all subjects and therefore treated episodes as a random factor. (It should be noted, however, that episodes were not selected randomly.) In the following summary of the analyses for both experiments, the F statistics based on subjects treated as a random factor are presented first, followed by the $F$ statistics based on episodes treated as a random factor.

Table 2 presents the RT means for the true responses (based on subjects treated as a random factor), as well as the error means. The variables for the true responses were the stimulus modality of the probe (picture or sentence), the explicitness of the probe (explicit or implicit), and congruency. The level of the later variable was considered the "same" if the probe event was in the same modality (picture or sentence) as its episode; otherwise it was considered "opposite." The analyses revealed faster $\mathrm{RT}$ to picture than to sentence probes $[\mathrm{F}(1,31)=107.09, \mathrm{p}<.001, \mathrm{SE}=72.1$, and $\mathrm{F}(1,7)=$ $74.66, \mathrm{p}<.001, \mathrm{SE}=46.6]$ and revealed faster $\mathrm{RT}$ to same than to opposite probes $[F(1,31)=14.99, p<.001$, $\mathrm{SE}=31.7$, and $\mathrm{F}(1,7)=7.61, \mathrm{p}<.03, \mathrm{SE}=20.1]$. The analyses revealed some evidence for an interaction between probe stimulus modality and explicitness $[F(1,31)=6.71, p<.02, S E=34.6$, but $F(1,7)<1]$, reflecting an advantage of explicits over implicits for picture probes but not for sentence probes. The interaction (based on subjects treated as a random factor) was examined separately for same and opposite probes, and the results indicated that only the same-modality probes showed the interaction $[\mathrm{F}(1,31)=7.03, \mathrm{p}<.02, \mathrm{SE}=$ $38.5]$; the opposite-modality probes did not $[F(1,31)<$ 1]. That is, RT was faster to explicit same picture probes than to implicit same picture probes but about the same to explicit same sentence probes as to implicit same sentence probes. The analyses also revealed some evidence for an interaction between probe stimulus modality and congruency $[\mathrm{F}(1,31)=6.60, \mathrm{p}<.02, \mathrm{SE}=35.7$, and $F(1,7)=4.56, p<.07, S E=25.7]$, reflecting a greater advantage of sames over opposites for picture probes than for sentence probes. Finally, the analyses revealed strong evidence of an interaction between explicitness and congruency $[\mathrm{F}(1,31)=6.50, \mathrm{p}<.02$, $\mathrm{SE}=46.5$, and $\mathrm{F}(1,7)=19.66, \mathrm{p}<.003, \mathrm{SE}=18.4]$,

Table 2

Mean RTs (in Milliseconds) and Error Percentages (EP) for Correct Responses to True Probes in Experiment 1

\begin{tabular}{|c|c|c|c|c|c|c|c|c|}
\hline \multirow[b]{3}{*}{ Modality } & \multicolumn{4}{|c|}{ Picture Probes } & \multicolumn{4}{|c|}{ Sentence Probes } \\
\hline & \multicolumn{2}{|c|}{ Explicit } & \multicolumn{2}{|c|}{ Implicit } & \multicolumn{2}{|c|}{ Explicit } & \multicolumn{2}{|c|}{ Implicit } \\
\hline & RT & EP & DT & $\mathbf{E P}$ & RT & EP & RT & EP \\
\hline Opposite & 1625 & 7.3 & 1529 & 7.5 & 2125 & $\begin{array}{l}7.8 \\
9.9\end{array}$ & 1970 & $\begin{array}{l}7.0 \\
7.6\end{array}$ \\
\hline
\end{tabular}


reflecting an advantage of sames over opposites for explicit probes but not for implicit probes. Follow-up tests confirmed this interpretation of the interaction: For explicit probes, RT was much faster to sames than to opposites $[\mathrm{F}(1,31)=20.91, \mathrm{p}<.001, \mathrm{SE}=37.3$, and $\mathrm{F}(1,7)=25.11, \mathrm{p}<.01, \mathrm{SE}=21.8]$, but for implicit probes, there was virtually no $\mathrm{RT}$ difference between same and opposite probes $[F(1,31)<1$ and $F(1,7)<1]$. An unexpected result was that RT to explicit opposites appeared to be greater than $\mathrm{RT}$ to other probes requiring a true response. A post hoc comparison (Scheffé test) revealed that this difference was significant $[F(1,31)=$ $12.87, \mathrm{p}<.05, \mathrm{SE}=33.6$, and $\mathrm{F}(1,7)=4.76, \mathrm{p}<.08$, $\mathrm{SE}=24.1]$. No other main effects or interactions were significant.

The variables for the false responses were relatedness (related sames, related opposites, or unrelated) and probe stimulus modality (picture or sentence). Table 3 presents the RT means for the false responses (based on subjects treated as a random factor) and the error means. In order to conduct an analysis that treats episodes as a random factor, unrelated distractors were arbitrarily assigned to episodes, one picture and one sentence unrelated event per episode. The analyses revealed faster RT to picture than to sentence probes $[F(1,31)=$ $137.37, \mathrm{p}<.001, \quad \mathrm{SE}=61.2, \quad$ and $\mathrm{F}(1,7)=43.38$, $\mathrm{p}<.001, \mathrm{SE}=49.0]$, and that RT was fastest to unrelated distractors and slowest to related opposite distractors $[F(2,62)=51.92, \mathrm{p}<.001, \mathrm{SE}=37.7$, and $\mathrm{F}(2,14)=23.18, \mathrm{p}<.001, \mathrm{SE}=29.0] . \quad$ A follow-up test confirmed that RT was faster to related sames than to related opposites $[\mathrm{F}(1,31)=11.29, \mathrm{p}<.02, \mathrm{SE}=$ 40.1 , and $\mathrm{F}(1,7)=8.75, \mathrm{p}<.03, \mathrm{SE}=26.0]$. However, if a comparison between same and opposite probes is done separately for those false related items that depict a change in a character or object in an explicit event or that contradict an explicit event (substitutions) and for those that depict an event that could have conceivably occurred in the setting of the episode but did not happen to occur (alternatives), then there is an advantage of sames over differents for substitutions [ $\mathrm{t}(17)=$ $2.09, \mathrm{p}<.05]$, but not for alternatives $[\mathrm{t}(13)=.283$, $\mathrm{p}>.30]$.

\section{Discussion}

As expected, RT was faster to explicit same probes than to explicit opposite probes, presumably because the

Table 3

Mean RTs (in Milliseconds) and Error Percentages (EP) for Correct Responses to False Probes in Experiment 1

\begin{tabular}{clccccc}
\hline & & \multicolumn{2}{c}{ Picture Probes } & \multicolumn{2}{c}{ Sentence Probes } \\
\cline { 3 - 4 } Probe & Modality & RT & EP & & RT & EP \\
\hline Related & Same & 1520 & 8.4 & & 2116 & 7.8 \\
Related & Opposite & 1655 & 6.7 & & 2253 & 9.8 \\
Unrelated & & 1293 & 5.1 & & 1855 & 4.9 \\
\hline
\end{tabular}

information in an explicit same probe matches both the surface and the conceptual representations of its target episode, whereas the information in an explicit opposite probe matches only the conceptual representation. As predicted by the conceptual identity hypothesis, but contrary to the conceptual nonidentity hypothesis, RT to implicit sames was virtually identical to the RT to implicit opposites. The explanation, according to the conceptual identity hypothesis, is that the information in an implicit probe is matched only to the conceptual representation of its episode and that representation retains nothing of the linguistic or physical qualities of the episode. Rather, conceptual memory appears to be a truly abstract level of thought, capable of representing the conceptual ideas of pictures and prose in equivalent forms. These results thus support the assumption of several theorists that the underlying meaning of discourse is abstract and language-free (J. R. Anderson, 1976; J. R. Anderson \& Bower, 1973; Norman \& Rumelhart, 1975; Schank, 1972).

The results of this experiment are inconsistent with another alternative that may be called the nonconceptual hypothesis. According to it, subjects store only verbatim information during the study phase, and no conceptual information at all. Any conceptual information needed is generated when probes are presented. Such a hypothesis would predict faster RT to explicit sames than to any other true probes. However, the nonconceptual hypothesis is unlikely for several reasons: First, it would predict that explicit sames would always be evaluated faster than any implicit probe, yet in the present experiment, implicit same sentence probes were actually evaluated slightly faster than explicit same sentence probes. Also, Keenan and Kintsch (1974) and McKoon and Keenan (1974) found essentially no explicit-implicit RT difference with verbal materials at delays on the order of minutes, and Baggett (1975) found, after a delay of $72 \mathrm{~h}$, no explicit-implicit RT difference with pictorial materials very similar to those used in the present study. Second, the nonconceptual hypothesis is unlikely because it claims that subjects either ignore or quickly forget the conceptual meaning of episodes but retain their surface features. Such a claim goes against research that shows that conceptual memory persists longer than surface memory (Bransford et al., 1972; Bransford \& Franks, 1971; Sachs, 1967). Finally, one version of the nonconceptual hypothesis might propose that, since subjects prefer to store only surface representations, the conceptual representations they generate when probes are presented contain features specific to the stimulus modality of the episode. If so, then this version, like the nonidentity hypothesis, erroneously predicts an advantage of implicit sames over implicit opposites. However, if the conceptual representation generated when probes are presented is abstract and contains no features specific to the stimulus modality, then the nonconceptual hypothesis, like the conceptual 
identity hypothesis, would correctly predict that RT should be the same to implicit opposites as to implicit sames. This second version of the nonconceptual hypothesis differs from the conceptual identity hypothesis only with respect to the issue of when conceptual information about an episode is generated. However, for reasons discussed above, it seems unlikely that subjects would wait until probes are presented before they generate ideas about the meaning of an episode.

The experiment also reveals a number of other interesting trends. The RT to true probes demonstrates that picture probes can be evaluated faster than sentence probes, probably because pictures can be encoded into memory faster than verbal materials (Friedman \& Bourne, 1976; Potter \& Faulconer, 1975). Also, the true RT data show that for both picture and sentence probes, RT is longer to explicit opposites than to any other true probe. This result is puzzling, but one possible explanation is that because explicit opposite probes contain conceptual information that is close but not identical to the conceptual information in some explicitly presented event, subjects may have suspected that some essential feature of the original event had been changed in the explicit opposite probe (perhaps to fool them) and therefore may have done more conceptual processing of explicit opposites. Finally, as mentioned above, the true RT data show that explicit sames are evaluated faster than implicit sames for picture but not for sentence probes. A possible reason may be that subjects generate more inferences during the study of prose episodes than during the study of picture episodes, and this additional conceptual information allows them to make faster judgments about implicit probes based on those prose episodes, thereby overcoming any potential advantage for explicit same sentences provided by the memory of the surface qualities of a prose episode. It is not clear, however, why subjects should generate more inferences while reading prose than while studying pictures, but perhaps the reason is that school experiences provide practice generating inferences while reading textbooks. Another possible explanation for the explicit same over implicit same advantage for picture but not for sentence probes might be that the surface representation of pictures is more durable or distinctive than the surface representation of prose. Such an explanation would also correctly predict a reduction in the explicit same/explicit opposite difference for sentence as opposed to picture probes, but it would incorrectly predict a reduction or elimination of a related same/related opposite difference for false sentence probes but not for false picture probes.

The data on the false probes demonstrated that false unrelated probes are judged to be false more rapidly than are false related probes, probably because RT to judge a stimulus to be false is slowed down any time a false probe resembles a target (e.g., Smith, Rips, \& Shoben, 1974). Finally, the false RT data show that RT to related same probes is faster than to related opposite probes, but only for related falses that depict contradictions or substitutions; for alternative-track events, there was no same-opposite RT difference. Samemodality contradictions and substitutions may be rejected rapidly relative to their opposite-modality counterparts because an obvious surface difference between such same-modality probes and explicitly presented events readily suggests a conceptual change. So, for example, a false probe that contains a waitress instead of a waiter but is otherwise identical to an explicitly presented event readily signals the conceptual basis for making a false judgment. Thus only minimal conceptual processing would be required to reject such a probe relative to its opposite-modality counterpart, for which there is no obvious surface change, since opposite probes are in the modality opposite that of their episodes. On the other hand, same-modality alternative-track falses depict no simple surface discrepancy that readily suggests the basis upon which the probe is to be judged false. Rather, subjects are required to do more conceptual processing of both same and opposite probes in an attempt to determine whether a plausible causal chain can be constructed to connect the probe with its episode. Thus alternative-track falses are likely to be processed like true inferences, and, according to the conceptual identity hypothesis, no same-opposite difference is expected for such false probes.

To recapitulate, the results of the first experiment are consistent with the conceptual identity hypothesis that the conceptual memory for picture episodes is identical in form to the conceptual memory for prose episodes, making conceptual memory a very abstract, language-free kind of mental representation. Two alternative hypotheses, a conceptual nonidentity hypothesis that proposes a conceptual representation that retains some modality-specific features and a nonconceptual hypothesis that proposes that subjects remember only surface features, were found to be inconsistent with the data. In addition, the results of the experiment showed that there was an explicit same over implicit same advantage for picture but not for sentence probes, possibly because prose episodes prompt more inferential processing than do picture episodes or because the surface representation of pictures is more durable than that of prose.

\section{EXPERIMENT 2}

The second experiment focused on two models of how conceptual information in a probe is compared with conceptual information in the memorial representation of an episode. The first is a simple scanning model that proposes that subjects scan each conceptual unit or fact stored with an episode in order to find a match between the fact and the conceptual information in a probe. An 
implicit probe might be matched to a fact by conducting a test to determine whether the probe is a logical inference of the stored fact. One important prediction of the scanning model is that RT to reach a decision about a probe should increase as the length of the episode increases, since longer episodes have more to-be-scanned facts.

The second model is a direct-access model that proposes that subjects do not scan lists of facts until they come upon a match; rather, they directly access the location or slot in memory where the relevant information for determining the truth value of a probe is stored. One mechanism for accomplishing direct access is based on schema theory (R. C. Anderson, 1977; Bobrow \& Norman, 1975; Minsky, 1975; Schank \& Abelson, 1977), which proposes that information pertaining to some commonly occurring situation activates a schema that represents, in a kind of abstract outline form, the prototypical situation. The events of any given episode are bound to the relevant slots in the schema. One function of a schema may be to direct memory search directly to those slots in which relevant information is stored. So, for example, if given a probe that depicts a short-haired man leaving a barbershop, subjects need not scan all the facts pertaining to the barbershop episode; they can restrict their search to the slot in the schema that pertains to exiting from the barbershop. In addition, much of the implicit information in an episode may be derivable from what is stored explicitly in the schema. An important prediction of the direct-access model is that RT to evaluate a probe should not increase as the length of the episode increases, since the schema into which an episode is assimilated can guide retrieval directly to the slot that explicitly contains the relevant information or provides an anchor for the constructed set of causal relationships between the information in the slot and the implicit probe.

The procedure of the second experiment was nearly identical to that of the first experiment, except that, in Experiment 2, the length of the episodes was varied. This procedure thus allows a test of the scanning and direct-access models and provides a second test of the conceptual identity hypothesis.

\footnotetext{
Method

Subjects. Thirty-two subjects from the same subject pool used in Experiment 1 participated in Experiment 2.

Stimuli and Apparatus. The stimuli and apparatus were the same as those used in Experiment 1.

Procedure. The procedure was generally similar to Experiment 1 , except for the following changes. In the study phase of the experiment, half of the episodes consisted of two events and the rest consisted of four events. The construction of a twoevent episode for Experiment 2 was accomplished by withholding the filler events of Experiment 1 from the study and test phases of Experiment 2. The explicit events of a two-event episode were selected to insure that subjects understood the stereotypic situation to which the episode referred. Table 1 indicates, for three of the episodes, the events that were paired
}

to construct twoevent episodes. In addition to the counterbalancing done in the first experiment, another counterbalancing was done to insure that, actoss subjects, each episode was presented as often as a short episode (two events in length) as it was presented as a long episode (four events in length).

\section{Results}

The principal data are again mean RT in milliseconds. The RT analysis includes only correct responses; the mean error rate over subjects was $7.5 \%$, with a range of $3.5 \%$ to $15.5 \%$. In general, errors were positively correlated with RT within each variable. Aberrant RTs, defined as those that were over 3 standard deviations larger than the overall subject mean RT, were excluded from the data analysis (this exclusion was less than $2 \%$ ), as were data from the practice trials.

Table 4 presents the RT means for the true responses (based on subjects treated as a random factor), as well as error means. Separate analyses were done on the picture and sentence data in order to simplify the analyses and to avoid the evaluation of a rather large number of irrelevant interactions. The variables for the response to the true probes were explicitness of the probe (explicit or implicit), congruency (same or opposite), and the length of the episode of the probe (short or long). The analyses on the picture probes requiring a true response revealed faster RT to explicit than to implicit probes $[\mathrm{F}(1,31)=11.26, \mathrm{p}<.002, \mathrm{SE}=62.0$, and $F(1,7)=8.01, p<.03, S E=42.9]$, faster $R T$ to same than to opposite probes $[F(1,31)=19.00, p<.001$, $\mathrm{SE}=59.6$, and $\mathrm{F}(1,7)=5.91, \mathrm{p}<.05, \mathrm{SE}=42.1]$, and some evidence for a tendency for RT to be faster to probes from long episodes than to probes from short episodes $[F(1,31)=3.80, p<.06, \quad S E=51.7$, but $\mathrm{F}(1,7)=1.30, \mathrm{p}<.30, \mathrm{SE}=72.8]$. Twenty-four subjects responded faster, on the average, to picture probes from long episodes ( $p<.05$ by a sign test). The analyses also revealed an interaction between explicitness and congruency $[\mathrm{F}(1,31)=6.61, \mathrm{p}<.02, \mathrm{SE}=78.0$, and $F(1,7)=9.75, p<.02, S E=43.9]$, reflecting faster $R T$ to sames than to opposites on explicit trials, but virtually no difference between sames and opposites on implicit trials. Follow-up tests confirmed this interpretation of

Table 4

Mean RTs (in Milliseconds) and Error Percentages (EP) for Correct Responses to True Probes in Experiment 2

\begin{tabular}{|c|c|c|c|c|c|c|c|c|}
\hline \multirow{3}{*}{$\begin{array}{l}\text { Epi- } \\
\text { sode }\end{array}$} & \multicolumn{4}{|c|}{ Picture Probes } & \multicolumn{4}{|c|}{ Sentence Probes } \\
\hline & \multicolumn{2}{|c|}{ Explicit } & \multicolumn{2}{|c|}{ Implicit } & \multicolumn{2}{|c|}{ Explicit } & \multicolumn{2}{|c|}{ Implicit } \\
\hline & $\mathrm{RT}$ & $\mathrm{EP}$ & RT & EP & RT & EP & RT & EP \\
\hline & \multicolumn{8}{|c|}{ Same Modality } \\
\hline \multirow{3}{*}{$\begin{array}{l}\text { Short } \\
\text { Long }\end{array}$} & 1325 & 5.5 & 1606 & 8.8 & 1945 & 7.9 & 1923 & 7.3 \\
\hline & 1 & 4.3 & 15 & 8.4 & 1948 & 4.7 & 1933 & 7.8 \\
\hline & \multicolumn{8}{|c|}{ Opposite Modality } \\
\hline Sho & 1655 & 7.6 & 16 & 9.4 & 2252 & 10 . & 19 & 9.0 \\
\hline Long & 1610 & 8.2 & 1562 & 6.4 & 2148 & 9.2 & 1885 & 6.8 \\
\hline
\end{tabular}


the interaction: On explicit trials, RT was faster to sames than to opposites $[F(1,31)=21.75, p<.001$, $\mathrm{SE}=70.0$, and $\mathrm{F}(1,7)=18.93, \mathrm{p}<.01, \mathrm{SE}=41.4]$, but on implicit trials, there was essentially no RT difference between same and opposite probes $[F(1,31)<1$ and $F(1,7)<1]$. No other interactions were significant. Since, in Experiment 1, there was evidence that RT to explicit opposites was greater than RT to other true probes, a similar comparison was conducted on the picture probe RT, averaged over episode length. This comparison was only marginally significant $[\mathrm{F}(1,31)=3.80$, $\mathrm{p}<.08, \mathrm{SE}=58.2$, and $\mathrm{F}(1,7)=1.48, \mathrm{p}<.25, \mathrm{SE}=$ 43.5].

The analyses on sentence probes requiring a true response revealed some evidence for $\mathrm{RT}$ to be faster to implicit than to explicit probes $[F(1,31)=10.90$, $\mathrm{p}<.002, \mathrm{SE}=58.5$, but $\mathrm{F}(1,7)=1.02, \mathrm{p}<.50, \mathrm{SE}=$ 74.6], and that RT was faster to same than to opposite probes $[F(1,31)=15.76, p<.001, S E=47.7$, and $\mathrm{F}(1,7)=4.28, \quad \mathrm{p}<.07, \quad \mathrm{SE}=51.0]$. There was no evidence of an overall effect of episode length, but there was some evidence of an Episode Length by Congruency interaction $[F(1,31)=3.80, p<.06, S E=$ 60.1 , and $F(1,7)=3.26, p<.12, \mathrm{SE}=24.5$ ], refelcting a tendency for RT to be faster to probes from long episodes than to probes from short episodes for opposite probes but for RT to be about the same to probes from long episodes as to probes from short episodes for same probes. Finally, the analyses revealed an interaction between explicitness and congruency $[F(1,31)=$ 4.82, $\mathrm{p}<.04, \mathrm{SE}=70.3$, and $\mathrm{F}(1,7)=7.48, \mathrm{p}<.03$, $\mathrm{SE}=28.1$, reflecting the fact that RT to sames was faster than RT to opposites for explicit but not for implicit probes. Once again, follow-up tests confirmed this interpretation of this interaction: For explicit probes, RT was faster to sames than to opposites $[F(1,31)=7.15, p<.03, S E=65.5$, and $F(1,7)=9.56$, $\mathrm{p}<.03, \mathrm{SE}=27.3]$, but for implicit probes, there was essentially no RT difference between same and opposite probes $[F(1,31)<1$ and $F(1,7)<1]$. No other interactions were significant. There was evidence that RT to explicit opposites was greater than RT to other true probes (averaged over episode length) $[\mathrm{F}(1,31)=10.98$, $\mathrm{p}<.01, \mathrm{SE}=68.4$, and $\mathrm{F}(1,7)=8.51, \mathrm{p}<.03, \mathrm{SE}=$ 35.2].

Table 5 presents the RT means for the false responses (based on subjects treated as a random factor) and error means. The variables for the false responses were congruency (same or opposite) and episode length (short or long). The unrelated probes were not included in the analyses, in order to investigate the effects of length; however, a t test revealed that for both picture and sentence probes, RT to unrelated falses was faster than RT to related falses [for pictures, $t(31)=4.96, p<.001$; for sentences, $t(31)=7.14, p<.001]$. The analyses revealed no significant effects for the picture data and revealed faster $\mathrm{RT}$ to same than to opposite probes for
Table 5

Mean RTs (in Milliseconds) and Error Percentages (EP) for Correct Responses to False Probes in Experiment 2

\begin{tabular}{|c|c|c|c|c|c|c|}
\hline \multirow[b]{2}{*}{ Probe } & \multirow[b]{2}{*}{ Modality } & \multirow[b]{2}{*}{ Episode } & \multicolumn{2}{|c|}{$\begin{array}{l}\text { Picture } \\
\text { Probes }\end{array}$} & \multicolumn{2}{|c|}{$\begin{array}{c}\text { Sentence } \\
\text { Probes }\end{array}$} \\
\hline & & & RT & EP & RT & EP \\
\hline Related & Same & $\begin{array}{l}\text { Short } \\
\text { Long }\end{array}$ & $\begin{array}{l}1606 \\
1578\end{array}$ & $\begin{array}{l}8.5 \\
7.4\end{array}$ & $\begin{array}{l}2088 \\
2024\end{array}$ & $\begin{array}{l}7.6 \\
7.8\end{array}$ \\
\hline Related & Opposite & $\begin{array}{l}\text { Short } \\
\text { Long }\end{array}$ & $\begin{array}{l}1573 \\
1570\end{array}$ & $\begin{array}{l}7.4 \\
7.0\end{array}$ & $\begin{array}{l}2024 \\
2148\end{array}$ & $\begin{array}{l}7.8 \\
9.3\end{array}$ \\
\hline Unrelated & & & 1482 & 3.8 & 1918 & 5.6 \\
\hline
\end{tabular}

the sentence data $[F(1,31)=4.96, p<.04, S E=56.7$, and $\mathrm{F}(1,7)=4.43, \mathrm{p}<.07, \mathrm{SE}=29.5]$, but, as in Experiment 1, a comparison across stimulus modality between same and opposite probes done separately for substitutions and alternatives revealed an advantage of sames over opposites for substitution $[\mathrm{t}(17)=2.53$, $\mathrm{p}<.05]$, but not for alternatives $[\mathrm{t}(13)=-.08, \mathrm{p}>.40]$. For both picture and sentence probes, 22 subjects responded faster, on the average, to probes from long episodes (both ps $<.05$ by sign tests).

\section{Discussion}

The principal results of Experiment 1 were replicated in Experiment 2: RT was faster to explicit sames than to explicit opposites but the same to implicit sames as to implicit opposites, consistent with the conceptual identity hypothesis that the meaning of picture and prose episodes is stored in an identical format. The results also replicated some trends found in the true data of Experiment 1: RT to explicit same picture probes was faster than RT to implicit same picture probes, but RT to implicit same sentence probes was slightly faster than RT to explicit same sentence probes, and RT to explicit opposites was greater than RT to other true probes. A trend found in the false RT data of Experiment 1 was only partially replicated: RT to related same false probes was faster than to related opposites, but only for sentence probes and not for picutre probes. However, as in Experiment 1, RT was faster to related sames than to related opposites for substitutions but not for alternativetrack events for both picture and sentence probes.

The main purpose of Experiment 2 was to examine the effects of episode length on RT to probes in order to test the predictions of the scanning and direct-access models. The results supported the direct-access model: RT was not greater to probes from long episodes than to probes from short episodes; in fact, there was a tendency for RT to actually decrease to probes from long episodes. This tendency is the opposite of the usual fan-size effect (an increase in RT to probes based on larger sets of facts) found in many fact-retrieval experiments (J. R. Anderson, 1974, 1976; J. R. Anderson \& Bower, 1973; Lewis \& J. R. Anderson, 1976; Thorndyke \& Bower, 1974). However, the fan-size effect may be 
rather fragile (Moeser, 1979); for example, it appears that when sets of facts may be assimilated into an organizing schema, no fan-size effect occurs (Moeser, 1979; Smith, Adams, \& Schorr, 1978; Experiment 2 of the present research). The suggestion here is that the schema guides retrieval directly to the relevant slot in memory in which the information necessary to make the true-false decision is stored, and thus the schema protects retrieval from the potentially interfering effect of the presence of related facts in memory.

Although the direct-access model based on schema theory accounts for the lack of a fan-size effect, a scanning model based on schema theory can also be evoked. According to such a scanning model, subjects scan all the facts associated with a schema in memory, regardless of the number of events explicitly presented during the study phase. Thus the same number of facts would be scanned in memory for short and long episodes. However, this kind of scanning model demands that subjects scan a potentially very large number of facts. In fact, even simple schemata such as "eating at a restaurant" probably contain an almost unlimited number of facts in a continuum from very general levels of description ("eating the meal") to rather specific levels of description ("lifting the fork"), although Bower, Black, and Turner (1979) found that subjects asked to generate 20 events for such episodes tended to avoid describing very detailed events. The scanning model would also have to make allowances for the false items that depict alternative-track events, since such events may be in the schema. Such a scanning model would thus require some rather elaborate system of flags and tags to guide the scan, in order to avoid items that might lead to an incorrect response.

The scanning model based on schema theory also provides no clear explanation for the tendency for RT to actually decrease to probes from longer episodes, except to explicit same probes (and implicit same sentence probes), for which there appeared to be no effect for episode length. An explanation based on the direct-access model might be that slots near those accessed by explicit events presented during the study phase are easier to access during later retrieval (see Bower et al., 1979, Experiment 6, for a similar idea). Thus as more explicit events are presented during the study phase, more of the schema's slots become readily accessible during the test phase. Explicit same probes will always contact a portion of the schema that was previously accessed; therefore, there should be no retrieval advantage for explicit sames from long episodes. But other probes are more likely to contact an activated portion of their schema when they come from long episodes, and therefore RT to such probes should be reduced. Note that a similar kind of explanation will not work for the scanning model because long episodes would activate more slots, which would presumably be scanned with a greater likelihood than nonactivated slots, suggesting an increase in RT to probes from long episodes. Another possibility is that, for short episodes, subjects may simply have a less clear idea about what schema is appropriate for interpreting the events and therefore have more trouble judging inferences based on short episodes. However, two event episodes were constructed to make obvious the appropriate schema. At any rate, any difficulty in identifying the schema should be greater for prose than for pictorial episodes (which contain additional visual cues concerning the appropriate schema), yet the tendency for RT to decrease to implicits from long episodes was as great for probes based on pictorial episodes as for probes based on prose.

In summary, the present studies have provided evidence for two important characteristics of the memory of episodes. First, the conceptual representation of episodes appears to store the conceptual information from picture and prose episodes in an equivalent format, making conceptual memory very abstract and languagefree. Second, retrieving information from episodes is done, not by scanning all the events in the episode, but by restricting the memory search to the location in memory in which the relevant information is stored.

\section{REFERENCES}

Anderson, J. R. Retrieval of propositional information from long-term memory. Cognitive Psychology, 1974, 6, 451-474. ANDERSON, J. R. Language, memory, and thought. Hillsdale, N.J: Erlbaum, 1976.

Anderson, J. R., \& Bower, G. H. Human associative memory. Washington, D.C: Winston, 1973.

Ande Rson, R. C. Encoding processes in the storage and retrieval of sentences. Journal of Experimental Psychology, 1971, 91, 338-340.

Anderson, R. C. The notion of schemata and the educational enterprise. In R. C. Anderson, R. J. Spiro, \& W. E. Montague (Eds.), Schooling and the acquisition of knowledge. Hillsdale, N.J: Erlbaum, 1977.

BaggetT, P. Memory for explicit and implicit information in picture stories. Journal of Verbal Learning and Verbal Behavior, $1975,14,538-548$.

Begg, I., \& Paivio, A. Concreteness and imagery in sentence meaning. Journal of Verbal Learning and Verbal Behavior, $1969,8,821-827$.

Bobrow, D. G., \& Norman, D. A. Some principles of memory schemata. In D. G. Bobrow \& A. Collins (Eds.), Representation and understanding. New York: Academic Press, 1975.

Bower, G. H., BlaCk, J. B., \& TURNER, T. J. Scripts in memory for text. Cognitive Psychology, 1979, 11, 177-220.

Bransford, J., Bahclay, R., \& Franks, J. Sentence memory: A constructive versus interpretive approach. Cognitive Psychology, 1972, 3, 193-209.

Bransford, J. D., \& Franks, J. J. The abstraction of linguistic ideas. Cognitive Psychology, 1971, 2, 331-350.

Friedman, A., \& Bourne, L. E. Encoding the levels of information in pictures and words. Journal of Experimental Psychology. General, 1976, 2, 169-190.

Harris, R. J., \& Monaco, G. E. Psychology of pragmatic implication: Information processing between the lines. Journal of Experimental Psychology: General, 1978, 1, 1-22.

Hayes-Roth, B., \& Hayes-Roth, F. The prominence of lexical information in memory representations of meaning. Journal of Verbal Learning and Verbal Behavior, 1977, 16, 119-136. 
Honeck, R. P. Interpretive vs. structural effects on semantic memory. Journal of Verbal Learning and Verbal Behavior, $1973,12,448-455$.

Johnson, M. K., Bransford, J. D., \& Solomon, S. Memory for tacit implications of sentences. Journal of Experimental Psychology, 1973, 98, 203-205.

KeEnan, J. M., \& KInTSCH, W. The identification of explicitly and implicitly presented information. In W. Kintsch (Ed.), The representation of meaning in memory. Hillsdale, N.J: Erlbaum, 1974.

King, D. R. W., \& Greeno, J. G. Invariance of inference times when information was presented in different linguistic formats. Memory \& Cognition, 1974, 2, 233-235.

KINTSCH, W. The representation of meaning in memory. Hillsdale, N.J: Erlbaum, 1974.

KINTSCH, W., \& Monk, D. Storage of complex information in memory: Some implications of the speed with which inferences can be made. Journal of Experimental Psychology, 1972, 94, 25-32.

Lewis, C. H., \& Anderson, J. R. Interference with real world knowledge. Cognitive Psychology, 1972, 8, 311-335.

McKoon, G., \& KeEnan, J. M. Response latencies to explicit and implicit statements as a function of the delay between reading and test. In W. Kintsch (Ed.), The representation of meaning in memory. Hillsdale, N.J: Erlbaum, 1974.

Minsky, M. A framework for representing knowledge. In P. Winston (Ed.), The psychology of computer vision. New York: McGraw-Hill, 1975.

Moeser, S. D. The role of experimental design in investigations of the fan effect. Journal of Experimental Psychology: Human Learning and Memory, 1979, 5, 125-134.

Norman, D., \& Rumelhart, D. Explorations in cognition. San Francisco: Freeman, 1975.

Pezde K, K. Cross-modality semantic integration of sentence and picture memory. Journal of Experimental Psychology: Human Learning and Memory, 1977, 3, 515-524.

Potter, M. C., \& F Aulconer, B. A. Time to understand pictures and words. Nature, $1975,253,437-438$.
PotTs, G. R. Information processing strategies used in encoding linear orderings. Journal of Verbal Learning and Verbal Behavior, 1972, 11, 727-740.

Pylyshy, Z. W. What the mind's eye tells the mind's brain: A critique of mental imagery. Psychological Bulletin, 1973, 80, 1-24.

Rosenberg, S., \& Simon, H. A. Modeling semantic memory: Effects of presenting semantic information in different modalities. Cognitive Psychology, 1977, 9, 293-325.

SACHS, J. D. S. Recognition memory for syntactic and semantic aspects of connected discourse. Perception \& Psychophysics, $1967,2,437-442$.

Schank, R. C. Conceptual dependency: A theory of natural language understanding. Cognitive Psychology, 1972, 3, 552-631.

Schank, R. C. The structure of episodes in memory. In D. Bobrow \& A. Collins (Eds.), Representation and understanding. New York: Academic Press, 1975.

Schank, R. C., \& Abelson, R. P. Scripts, plans, goals, and understanding. Hillsdale, N.J: Erlbaum, 1977.

Smith, E. E., Adams, N., \& Schorr, D. Fact retrieval and the paradox of interference. Cognitive Psychology, 1978, 10, 438-464.

Smith, E. E., Rips, L. J., \& Shoben, E. J. Semantic memory and psychological semantics. In G. H. Bower (Ed.), The psychology of learning and motivation (Vol. 8). New York: Academic Press, 1974.

Thorndyke, P. W. The role of inferences in discourse comprehension. Journal of Verbal Learning and Verbal Behavior, 1976, 15, 437-446.

Thorndyke, P. W., \& Bower, G. H. Storage and retrieval processes in sentence memory. Cognitive Psychology, 1974, 6, 515-543.

Yuille, J. C., \& Paivio, A. Abstractness and the recall of connected discourse. Journal of Experimental Psychology, $1969,82,467-471$.

(Received for publication October 24, 1979; revision accepted April 28, 1980.) 